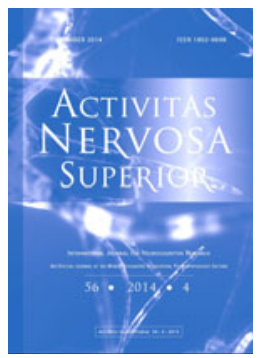

\title{
PROBABILITY FORECAST IN DIFFERENT TYPES OF BEHAVIOR AND IN REM SLEEP IN FRAME OF SEARCH ACTIVITY CONCEPT
}

\author{
Vadim Rotenberg* \\ Tel Aviv University, Bat-Yam, Israel
}

\begin{abstract}
The search activity concept proposed a new classification of the types of behavior according to the adaptation to the environment. In this article I have explained the story of the creation of this concept and discuss the probability forecasts of the outcomes of activity in different types of behavior and in REM sleep dreams. The direction of search can be changed if the outcome of activity does not correspond to the forecast. Stereotyped behavior uses habitual skills and algorithms with a definite forecast of the results. Chaotic (panic) behavior does not include monitoring of the results of activity. It determines the unreasonable fluctuations of the direction of activity independent of its outcome without probability forecast. Finally after painful failures it can lead to the renunciation of activity where the forecast of the positive results of activity is on the zero level. In REM sleep dreams subject is separated from the surrounding environment and the "failures" of the virtual activity are not dangerous for the subject. The process of activity by itself is more important than its direction and its outcome. For this reason search activity in dreams imitates chaotic activity in wakefulness however without its negative outcome and after awakening subject is ready to perform normal search activity.
\end{abstract}

Key words: Chaotic behavior; Depression; REM sleep; Search activity; Stress

\section{INTRODUCTION}

Before discussing the main topic of this theoretical article - the role of probability forecast in different types of behavior and in REM sleep - I would like to present the history of the development of the search activity concept that elucidates the solution of many contradictions related to this topic.

The initial step to the development of the Search Activity concept was done when 45 years ago I started to investigate sleep in patients with insomnia caused by neurotic anxiety and depression. I have suddenly found that after awakenings in REM sleep these patients present much less often dream reports than healthy subjects and their dream reports are less vivid and less rich according to images, events and actions. It was unexpected because previously it was already shown by some investigators that those healthy subjects who are more emotionally sensitive than other healthy subjects have more dream reports and these reports contain more

*Correspondence to: Vadim Rotenberg, email: vadir@post.tau.ac.il

Received November 15, 2014; accepted December 2, 2014; Act Nerv Super 56(4), 95-104; ISSN-1802-9698 
events and images. Patients suffering from neurotic disorders are more sensitive than even the most sensitive healthy subjects but they displayed opposite features of their dreams. I came to the hypothesis that their REM sleep is possibly functionally not efficient and it may play an important role in the pathogenesis of their mental disorders.

But what exactly is the essence of this non-efficiency? Dreams of humans were traditionally considered by psychologists of the psychoanalytical school as a mental state that represents inner motivational conflicts and present to the suppressed motives an opportunity to be discharged in dreams or to be integrated with acceptable motives in the virtual reality of dreams. It was possible to propose that this function is lost in patients and it determines the increase of repression as a defense mechanism that causes anxiety. It was really shown in some investigations (Grieser et al., 1972) that REM sleep deprivation in healthy subjects causes repression. However, the problem was that REM sleep is an important part of sleep not only in humans but also in highly developed animals that have not neither the inner motivational conflicts, nor psychological defense mechanisms. On the other hand, REM sleep deprivation in animals also caused the rebound effect of this sleep stage and being prolonged such deprivation disturbed the animals' waking behavior. I come to the conclusion that it is reasonable to search for some common functions of REM sleep in animals and humans.

At that time it was already known that in animals REM sleep is characterized by the synchronized hippocampal theta- rhythm. This rhythm is present also in wakefulness and behavior in wakefulness accompanied by this rhythm is very polymorphic. It was aggression and defense reactions (fight and flight), orientation in the new environment, self-stimulation of the particular brain zones. It was difficult to find a common characteristic and essence of these types of behavior. They were characterized also by opposite emotional feelings: selfstimulation is accompanied by positive emotions and fight and flight represent negative emotions. Fight, orienting behavior and self-stimulation display an approach behavior while flight displays an avoidance behavior.

\section{INSIGHT OF SEARCH ACTIVITY}

I was overwhelmed by all these contradictions and suddenly got an insight. I understood that it is something in common between all these forms of behavior, and it is what I called search activity. By search activity is understood an active behavior (overt or covert, mental) that is oriented on changing the situation and environment or on changing the subject's attitude to it, with uncertainty regarding the possible results of this activity but with the constant monitoring of the results at all stages of activity (see in details Rotenberg, 2009; Rotenberg, 2012).

During the orienting behavior animal is searching what is going on in the environment. It can be performed without active overt behavior, just on the mental level. In the process of fighting animal is searching how to win. During flight animal is searching how to avoid the dangerous situation and to safe itself. Self-stimulation also corresponds to the notion of search activity: animal has to correct the process of self-stimulation in order to avoid the distribution of physiological activation on brain zones that have a negative outcome on self-feeling. Thus animal regularly stops self-stimulation and starts from the beginning after a short delay.

When I came to this notion of search activity as a general characteristic of behavior, I proposed that REM sleep in animals and in humans is characterized by the covered search activity in dreams that restores the potential of search in the subsequent wakefulness if for some reasons this activity decreased before sleep. I came to the conclusion that search activity has to play a crucial role in the process of the development of every member of the society and of the all society. The adaptation to the continuously changing and polydimensional environment and attempts of the adaptation of the environment by itself to the subject's needs would be impossible without such activity performed without definite probability prognosis but with the continuous consideration of the results of this activity in order to change the 
direction of activity according to the achieved results and goals. On the other hand, such flexible activity requires a lot of mental skills and of energy for continuous efforts and can lead to the exhaustion if the process of search by itself would not accompanied by the restoration of subject's skills and energy. It means also that search activity as a process restores subject's forces and is important for the subject's resistance to stress and for the preservation of subject's mental and somatic health.

In different stressful conditions search activity may decrease after many failures and may be displaced by the renunciation of search (giving up). According to my hypothesis it can lead to the diseases. It means that humans and high animals need a natural biological mechanism that can compensate this outcome of stress and restore search activity after giving up. I have made a proposition that it is the process of the particular search activity in REM sleep dreams that restores this activity in the subsequent wakefulness. When I came to this point, I understood that this theory will be estimated by scientists as a pure speculation if it would be not confirmed by the direct investigations performed on animals. I am not a physiologist and cannot perform such investigations. For some months I was thinking whom I can convince to do it.

\section{THE INVESTIGATIONS ON ANIMALS: BEHAVIOR AND HEALTH}

Suddenly the problem was solved by occasion. I have met a physiologist Victor Arshavsky who just before our meeting has on rats and rabbits investigated the dynamic of experimental pathological processes and artificial experimentally induced forms of somatic and nervous disorders (anaphylactoid edema; arrhythmia of cardiac contractions; epileptic seizures; extrapyramidal disturbances) in different emotional states. The electrodes were implanted in the experimental animals' brain in the lateral and ventromedial parts of the hypothalamus. Stimulation of lateral hypothalamus provoked self-stimulation (as a model of positive emotional experience) and stimulation of the ventromedial hypothalamus caused reaction of avoidance (as a model of negative emotional experience). The initial hypothesis of this investigation was that positive emotional state will block the artificial pathology while negative emotions will cause the exacerbation of them. This hypothesis corresponded to the main statement in psychophysiology at that period that positive emotions have positive and negative emotions have negative outcomes on health.

However, V. Arshavsky found that his investigations do not confirm this commonly accepted statement: pathological process sometimes decreased when animal displayed signs of negative emotional state, and sometimes increased during self-stimulation. The traditional psychophysiological approach does not explain such contradictions. According to my idea of search activity, I proposed not to concentrate attention on the emotional state by itself but to estimate the behavior of animals in different emotional states. It brought a definite solution of the problem. When animals displayed an active behavior (active avoidance, tendency toward fight or flight during brain stimulation or active self-stimulation) this behavior reduced the distinction of pathologic manifestations; freezing (a passive behavior with a high emotional tension) speeds a progress of the pathological process, and passive self-stimulation (when animal simply lay down on the pedal sending rare pulse bursts to the brain) at least did not decrease the development of the pathological process. The core of the effect of active behavior was also not in the motor activity by itself: it was shown in some investigations that when in order to avoid punishment animal has to inhibit motor activity arbitrary the successful "learners" had less gastrointestinal lesions than animals who continued active motor behavior (Goesling et al., 1974). During the orientation in new conditions motor activity of animal can also decrease (it can just quietly look around) without negative outcome for health. Thus search activity seemed to be the crucial factor in the prevention of somatic disturbances, especially in stressful conditions. 
The anti-homeostatic nature of search activity corresponds to the philosophical background of the physiology of activity proposed by N.A. Bernstein.

On the next stage of our collaboration with V. Arshavsky we have checked the hypothesis of the role of REM sleep and dreams in the restoration of search activity. We have studied the change of sleep structure of rats following the passive-defense reaction (freezing, renunciation of search) caused by the stimulation of the ventromedian nucleus of hypothalamus. Sleep was registered for several hours prior to the stimulation (several days after the implantation of electrodes) and just after the stimulation. After the stimulation the percentage of REM sleep increased almost twice (Rotenberg \& Arshavsky, 1979). The behavior of animals after this increase was normal and active. It was possible to conclude that the increase of REM sleep compensated the state of freezing. At the same time in many investigations was shown that self-stimulation and active defense reaction decreases total REM sleep duration as a sign of the decreased requirement in this stage after active behavior.

\section{INVESTIGATIONS ON HUMANS: STRESS AND SLEEP}

We have turned to the investigations on humans. We have investigated sleep structure of healthy students during the examination period, on the post-examination night, and of the same students on one of the post-holiday nights. Thirty minutes before the examination and thirty minutes after we have measured in students pulse frequency, arterial pressure and bioelectrical activity of the orbicular muscles of the mouth. The control data of these indices were obtained on one of the term days. Before the sleep and after the awakening on the postexamination night and on the control night students were asked to solve logical tasks of the Raven test.

Before the examination all subjects displayed a rise of autonomic and EMG indices compared to the control rest period what was a sign of the expected emotional tension. After the examination in some students (Group 1) these indices dropped to the control level while in other students (Group 2) they remained on the stable high level. What was interesting, the objective results of the examination were similar in both groups what means that students of both groups were prepared to the examination. The subjects of Group 2 demonstrated a significant increase in the percentage of REM sleep on the post-examination night compared to the control night and to the subjects of Group 1 on the post-examination night. It was also a relative decrease of delta-sleep in this group 2 in the post examination night (resembling the sleep disturbance in anxiety and depression) and a positive correlation between the total sleep duration and the proportion of REM sleep $(+0.74)$ and a negative correlation $(-0.6)$ between the duration of delta-sleep and of REM sleep.

We have come to the conclusion that students of both groups demonstrated the increased emotional tension before the examination but the quality of this tension was different. The members of Group 1 exhibited a normal, productive anxiety that contains search activity and ensured physiological and psychological mobilization in order to overcome the stressful situation of examination. It is quite expectable that after they successfully passed the examination this mobilization diminished and REM sleep was not increased. Students of Group 2 presumably were characterized by the opposite type of emotional tension, by neurotic anxiety that does not contain search activity and adaptive psychophysiological mobilization and does not help by itself to overcome stressful situation. Moreover, it makes it more difficult. Fortunately these students were good prepared to the examination, however even when the examination successfully past this success by itself does not reduce this maladaptive emotional tension that displays the state similar to freezing (renunciation of search). It requires increased REM sleep for its compensation, and indeed only after the postexamination night all autonomic signs of emotional tension decreased in these students. It is also an additional confirmation of this hypothesis: in comparison to students of Group 1, students of Group 2 showed worse results in resolving logical tasks on the evening after 
examination. These results improved after the post-examination night sleep. The nonproductive emotional tension that appears during and just after the examination may explain this difference. What is important, this emotional tension that is opposite to the emotional tension that includes search activity does not disappear by itself when the problem is solved, it needs REM sleep for its reduction. On the other hand, strong search activity during the waking behavior decreases the requirement in REM sleep in the subsequent sleep, and it may explain the decrease of REM sleep during the "first night effect", when subject started to sleep in a new environment. This new environment requires the orientation in it and a search for its essence, whether it is dangerous or not, and such search activity decreases the REM sleep pressure. The higher is search activity during the day, the less is requirement in REM sleep. Because the duration of REM sleep substantially determines the duration of the all night sleep, the strong search activity during the day may cause the decrease of sleep duration without any negative consequences for the subject, like it often happened in normal subjects so-called "short sleepers" who are very active during the day. The decreased sleep duration in creative people that are in the state of creative excitement can be explained in the same way - creation is a definite sign of search activity.

Many years ago I have investigated the night sleep of a young outstanding chess-player who later became very well known in the world. I investigated his sleep when he participated in one important chess-play. During the first day he has shared the first place with another participant what was very frustrating for him as a very ambitious person. His sleep after this day was long, like in long-sleepers, and contained around 30\% of REM sleep while delta-sleep was relatively reduced according to his age. On the second, final day of the game he was a winner, achieved a first place and afterward his sleep was short like in short-sleepers and contained much less REM sleep. I have made a conclusion that this person is very sensitive to the results of such games, may give up after failures and in such cases need REM sleep for the restoration of his search activity, and during the long game with failures his REM sleep may be unable to correspond to the requirement in it and it may be dangerous for his health. Actually some years later when he participated in such competitive game with another very strong chess-player after some failures he displayed somatic disorders.

As it is well known the need of REM sleep may increase in some mental disorders accompanied by the non-productive emotional tension like anxiety or depression. In these disorders REM sleep latency is often decreased due to such increased need, however these disorders by themselves usually causes the disturbance of sleep and are accompanied by the REM sleep dysfunction [as I have already shown when I presented data of my investigations of REM sleep dreams in mentally sick people (Rotenberg, 2009)]. As a result the increased need in REM sleep dreams cannot be satisfied and search activity is not restored.

While analyzing the results of the investigations, it is necessary to take into consideration that the functional insufficiency of REM sleep in mental and psychosomatic disorders is only a general tendency and takes place not in every night. We have shown, for instance (Indursky, Rotenberg, 1998) that even in patients with major depression mood in the morning after awakenings in almost $20 \%$ of all nights is better than in the evening (what is not typical for depression) and in such cases eye movement (EM) density in REM sleep has in average a normal dynamic: it increases from the first to the last REM sleep period what is also not typical for depression where often EM density is higher in the first cycles. In the rare cases in depression slow wave sleep displays a sudden increase in the last cycles (in normal sleep it always dominates in the first cycles) and in such exceptional cases this increase of slow wave sleep is regularly predicted by the increase of EM density in the previous REM sleep period. I suppose that such increase reflects the temporarily restoration of REM sleep function what is leading to the temporarily decrease of the maladaptive emotional tension/depression and it determines the sudden increase of slow wave sleep that is usually suppressed by this emotional tension. Search Activity Concept in details was presented in our publications (see Rotenberg \& Arshavsky, 1979; Rotenberg, 2009). 


\section{PROBABILITY FORECAST IN DIFFERENT TYPES OF BEHAVIOR}

Now let us turn to the second topic of this article. On the basis of the definition of search activity, I have proposed a new classification of behavior according to its adaptive function and attitudes towards the environment. Search activity as it was emphasized is an attempt to change the situation that does not satisfy subject (or to change the subject's own view on the situation) with uncertainty regarding the future results of this activity but with constant monitoring of these results at every stage of activity and is characterized by the indefinite probability prognosis. Subject has a goal - the unpleasant or frustrating situation must be changed or something attractive has to be achieved. However, first of all the goal by itself is often not very definite, for instance what exactly must be achieved in the process of creation or by what concretely has to be displaced the present undesired situation. Secondly, while making efforts to change the complicated situation subject cannot be sure about the outcomes of his/her efforts because many different factors in their dynamic interrelationships may influence this outcome. The prognosis of the outcome has to be flexible and subject must be ready to change it as well as the direction of his activity when in the process of search he/she is meeting something unpredictable. Search is search because its outcome is not definite. A prognosis is necessary on every stage of activity because it determines the direction of efforts. However this prognosis has not to be rigid in order to give subject an opportunity to take into consideration all new factors and events that appear in the process of behavior, and to change the direction of activity according to them.

Due to the probability forecast (prognosis) subject is able to compare the actual results of his activity with previously expected intention in order to correct the own expectations and behavior. It determines the reconsideration of the direction of search on the basis of previous and actual experience.

The concept of the probability prognosis that is not absolutely definite but has some reasons was proposed and developed in details by I.M. Feigenberg (2008) and it was a very important addition to the general theory of the activity of N.A. Bernstein (1996) that I have mentioned in the beginning of this article. The role of the probability forecast not only in search behavior but also in other types of behavior was not represented in a comprehensive way in my previous publications. For this reason I decided to speak about the probability forecast in more details.

However, there are some forms of behavior that are not based on the indefinite prognosis. Stereotyped behavior uses habitual skills and is based on a definite forecast of the results equal to 1 . Subject is trying to behave in the changing situation and condition according to the previous stereotypes. In many conditions that require to follow definite rules this behavior is adaptive. Stereotyped behavior with the definite forecast of the results of actions even when it promotes satisfaction of requirements (for instance, during the consummatory behavior) is not accompanied by hippocampal theta-rhythm.

Of course, all of us in our everyday life often behave in a stereotyped way when we are dealing with something that we estimate as very familiar and clear and what according to our experience (real or illusive) has always the same outcomes. For instance, after awakening in the morning we take our dresses that are often the same as yesterday; we are taking the breakfast and we are sure that we will have it, etc. Thus, it is a characteristic of behavior in a simple and relatively restricted condition. However, even the conditions for this familiar activity can suddenly change (dress is found to be dirty, electricity does not work). These conditions can also change as an outcome of our own behavior because we are not robots and our behavior is not the realization of the computerized program. In an open environment the probabilities are regularly changing in the process of activity and they cannot be determined in a stable way. The more complicated is our behavior and the more it depends on external events, on conditions that can suddenly appear or change, and especially on relationships with other people the less can be an objective prediction of the outcome of our activity. In such 
cases our behavior has to include an open search in order to be successful. However, there are people who according to the previous experience, bringing up process or to the peculiarity of their mentality and personality are more predisposed to the stereotyped behavior than to the search activity and are rigidly oriented on the definite prognosis of the outcome of the own activity.

Their behavior is not flexible, it is difficult for them to learn according to their own mistakes and to correct the direction of their activity. Of course, if everything is totally determined from the very beginning like mathematical tasks constructed according to definite rules stereotyped behavior is normal and adaptive. We often behave in an almost stereotyped way when the prognosis is really definite until something new and unpredictable appears. When it is necessary to correct behavior according to the unpredictable outcomes stereotyped behavior is no more adaptive and leads to failures. However, even when stereotyped behavior corresponds to the real conditions in the environment, it by itself, in opposite to search activity does not increase the body resistance to diseases and to stress and does not restores skills and energy, because it is related to the process of search.

Another type of behavior in wakefulness is maladaptive in any case although, paradoxically, on the first glance it may imitate search activity. It is chaotic activity (often represented during panic behavior). The probability forecast is on zero level. Subject spontaneously and without any reasons changes the direction of activity just before it is possible to make any conclusion about the possible outcome. The results of activity are not taken into consideration and its direction cannot be corrected. It determines an unreasonable fluctuation of activity, in opposite to search activity when the direction of activity can be changed only after the analysis whether it corresponds to the goals and is correct. Chaotic activity is pushed by the strong drive to change everything immediately due to the emotional tension. Every weak signal from the environment that has no real meaning may change the behavior because subject does not have a definite view on the situation and cannot estimate what is going on. Chaotic activity may be accompanied by the non-realistic intrinsic feeling that in the process of such active behavior some dangers can be avoided and a positive outcome can appear if subject is continuously running from one point to another. It is a mistake when we are speaking about behavior in a real environment that needs to be estimated. During chaotic activity dominates brain dopaminergic system (Rotenberg, 2009).

Definitely, such position that does not allow change the false direction of activity is leading to many real failures. After many painful failures chaotic activity often became displaced by the renunciation of any activity, to the state of giving up that not only makes subject helpless and hopeless in front of stressful events but also, as I have shown in the first part of this article, decreases body resistance and causes the development of mental and psychosomatic diseases. In this state subject has a negative prognosis of any outcome of his activity and of any future change of the situation. It creates a vicious circle - the absence of the hope to solve the problem blocks any attempts to change the situation and as result hope has no chance to appear at all.

It is combined with the negative estimation of all information that subject gets from outside like it happened in depression, and it increases the feeling of hopelessness and helplessness. Feigenberg (2008) proposed an active component of the probability forecast that determines the perception of environment and may play an important role in the development of active behavior as well as in renunciation of search. "You are getting from the world what you expected to get". Such expectation of the quality of important signals makes these signals subjectively more definite and strong even if they are objectively indefinite and weak.

On the other hand, if you expect from the very beginning or in the process of activity that you are unable to change the situation in the positive direction any information may be accepted subjectively as a confirmation that nothing can be done. Such attitude to the information from the environment blocks all attempts to search for the positive outcome. It is an influence of renunciation of search on the subjective estimation of everything what is going. 
In the process of search activity when subject is oriented on the active consideration and objective estimation of the conditions including all outcomes of subject's own behavior without illusions but with a hope to overcome obstacles based on the positive self-estimation, this active component of probability prognosis plays a positive role. In the process of search subject is oriented of course not only on signals that confirm such hope and is ready to react on all events, is open to any information. Search activity has to be flexible. However a prognosis related to the final goal of activity helps to understand signals correctly and to use them in the constructive way.

If subject is in a state of a strong emotional tension his probability prognosis determined by this tension may cause the alteration of the signals and disturbs their correct understanding. I suppose that it happened often when search activity is displaced by panic (chaotic activity) or by giving up (renunciation of search). However it can happen also when stereotyped behavior dominates with a tendency to interpret all information according to the very rigid monosemantic model. On the other hand, if in the process of search activity the information about the actual situation does not correspond to the initial prognosis or corresponds only partly to the goal of activity, subject does not ignore those aspects of information that are in contradiction with the positive prognosis but at the same time does not give up in front of this negative outcomes. The essence of search activity is that subjects use the unexpected outcomes to correct the direction of activity. At the same time, in the process of stereotyped activity this information can be ignored, and in the state of renunciation of search is often ignored the opposite information that can bring hope and presents an opportunity for the positive outcome if subject will make some efforts.

In the process of chaotic activity subject can suddenly and as if for occasion start to use the direction of activity that may lead to the success but afterward also suddenly and without any reasons, before getting a real feedback from this new way jumps into another direction being unable to extract any useful information from his previous activity and experience. Destructive emotional tension of panic may determine an irrelevant selection of signals from the general noise that has no meaning for adaptive behavior. Such selection is determined by subject's fear and expectation of something dangerous.

If the objective condition does not allow normal search activity while subject has a strong motivation to such activity it may cause mental disorders. For instance, sensory deprivation may cause hallucinations as an artificial search activity.

\section{PROBABILITY FORECAST IN REM SLEEP DREAMS}

Now let us turn to the mental activity in REM sleep. As it was shown, the functionally efficient REM sleep is represented by dream reports, and dreams restore search activity in the subsequent wakefulness. However it does not mean that all dreams by themselves contain search activity according to its definition, although in many dream contents it is possible to find signs of it. In such dreams, dreamer is changing the direction of his activity in the virtual world of dreams, sometimes according to the outcome of performed efforts. For instance, in my middle-age period I often had a dream that as a student I am preparing for the examination and have not enough time and feel myself lost and waited for the negative outcome. This dream appeared many times in my life usually in real stressful conditions. But once when I was inside of this dream I suddenly became very angry and exclaimed "But they have to take into consideration that I am already a Doctor of Science!". It was an active reaction, a search for going out of this situation and for avoiding helplessness, and what is interesting, this story with feeling of helplessness disappeared from my further dreams.

However often it does not happened and what is going on in dream resembles more chaotic activity: subject is jumping from one virtual story to another before he is finishing his fight with a previous obstacles and problems and without estimating the possible outcome of his virtual behavior. The dream tails exchange each other without regularity and some of them 
can be accompanied by failures. Nevertheless, in most cases these are normal dreams that do not lead to the feeling of hopelessness and the activity in dreams does not stop: subject starts a new fight as if from the very beginning. Only if activity in dream stops and the content of dreams decreases or even disappears, it is a sign of REM sleep insufficiency.

What is the reason of such difference between chaotic activity in wakefulness where it is maladaptive and in dreams where it is adaptive and restores search activity in the subsequent wakefulness? I suppose that it is an essential difference between virtual chaotic activity and real chaotic activity. In dreams subject is totally separated from the real environment and his virtual behavior is not dangerous for him and cannot lead to real failures. On every step he/she can start his virtual activity from the very beginning and its process by itself is more important than its outcome and its direction. It is like a game in special conditions that brings subject a feeling of excitement by itself. It does not matter whether the subject is a winner or not and until he/she is able immediately to forget about the metaphoric failures (like it happened in normal dreams - most of them disappear from our memory) and to start new attempts in another story the subject is protected from giving up and the p r o c e s of chaotic search helps to restore brain mechanisms of search activity.

Dream became functionally insufficient only when the process of this virtual search stops probably because it became overwhelmed by the state of giving up that started in wakefulness and continues in dreams with images and feelings of stable catastrophic failure. Instead of overcoming this state REM sleep dreams became suppressed by it. Another reason may be the weak imagination in dreams, because the process of the real or chaotic search in dreams is on the basis of images.

The present article displays an attempt to show how the integration of the search activity concept with the concept of probability prognosis opens a new way for analysis and explanation of different (adaptive and maladaptive) forms of behavior.

In addition to the meaning of the search activity concept for the theoretical consideration of the role of different types of behavior in adaptation to the stressful and flexible environment, it may be used as an important background for the creating of the new methods of treatment of mental and psychosomatic disorders. On the other hand, all tasks presented to the patient that can stimulate his/her active attitudes to the actual conditions increase self-estimation and belief that he/she is able to change these conditions and this searching of new ways may be very helpful for treatment. It would be a mistake to use only the relevant medications because even if these medications restore the activity of brain monoamines (noradrenaline, serotonine) that are necessary for search activity, subject needs real constructive goals and high estimation of self in order to realize this biological potential in the active adaptive behavior.

If psychotherapy does not provide subject's search activity with the relevant goals and directions, search activity may became socially maladaptive and destructive or it may lead to failures that finally, according to the negative feedback, may turn subject back to the renunciation of search or to chaotic behavior.

On the other hand, by taking into consideration the role of the functionally sufficient dreams in REM sleep for the restoration of search activity, and the important role of the right hemisphere image thinking and its flexibility in dream mentation, as well as the functional insufficiency of image thinking in almost all mental and psychosomatic disorders, it is necessary to restore the right hemisphere functions in the process of psychotherapy (Rotenberg, 2012).

\section{CONCLUSION}

The main statement of this article is that search activity in wakefulness is characterized by the indefinite probability forecast, while stereotyped activity is characterized by the definite forecast. On the other hand chaotic activity is characterized by the absence of any probability forecast. Renunciation of search is characterized by the definite negative forecast before the 
start of activity. REM sleep dreams are characterized by the chaotic search when the direction of activity is extremely flexible because it is a virtual activity where the process is much more important than the outcome of activity.

\section{REFERENCES}

Bernstein, N.A. (1996). On Dexterity and its development. L. Erlbaum Publ. Mahwah, New Jersey.

Feigenberg, I.M. (2008). The probability forecast in the human's activity and in the behavior of animals. Newdiamed, Moscow.

Goesling, W.J., Buchholz, A.R., \& Carreira C.J. (1974). Conditioned immobility and ulcer development in rats. Journal of General Psychology, 91, 231-236.

Grieser, C., Greenberg, R., \& Harrison, R. (1972). The adaptive function of sleep: Differential effects of sleep on dreaming and recall. Journal of Abnormal Psychology, 80, 280-286.

Indursky P., \& Rotenberg V.S. (1998). Change of mood during sleep and REM sleep variables. International Journal of Psychiatry in Clinical Practice, 2, 47-51.

Rotenberg, V.S. (2009). Search activity concept: Relationship between behavior, health and brain functions. Activitas Nervosa Superior, 51, 12-44.

Rotenberg V.S. (2012). Self-Image and Behavior. (Amazon)

Rotenberg, V.S., \& Arshavsky, V.V. (1979). REM sleep , stress and search activity. Waking and Sleeping, 3, 235-244. 